\title{
A Study of Online State-of-Health Estimation Method for In-Use Electric Vehicles Based on Charge Data
}

\author{
Di ZHOU ${ }^{\dagger, \dagger \dagger a)}$, Member, Ping FU ${ }^{\dagger}$, Hongtao YIN ${ }^{\dagger}$, Wei $\mathrm{XIE}^{\dagger \dagger \dagger}$, and Shou FENG ${ }^{\dagger}$, Nonmembers
}

\begin{abstract}
SUMMARY The real-time state-of-health ( $\mathrm{SOH}$ ) estimation of lithiumion batteries for electric vehicles (EV) is essential to EV maintenance. According to situations in practical applications such as long EV battery capacity test time, unavailability of regular daily tests, and availability of full-life-cycle charge data of EV recorded on the charging facility big data platform, this paper studies an online in-use EV state-of-health estimation method using iterated extended Gaussian process regression-Kalman filter (GPR-EKF) to incorporate lithium-ion battery data at the macro time scale and the micro time scale based on daily charge data of electric vehicles. This method proposes a kernel function GPR (Gaussian process regression) integrating neutral network with cycles to conduct fitting for data at the macro time scale to determine colored measurement noise; in addition, fragment charge data at the micro time scale is adjusted with real-time iteration to be used as the state equation, which effectively addresses issues of real-time SOC calibration and nonlinearization. The pertinence, effectiveness and real-time performance of the model algorithm in online battery state-of-health estimation is verified by actual data.

key words: state of health, fragment charge data, iterated GPR-EKF, inuse EV battery
\end{abstract}

\section{Introduction}

Traction batteries are the power source of electric vehicles. State of health $(\mathrm{SOH})$ of traction batteries is a critical parameter characterizing current states of EV batteries. Correct and real-time $\mathrm{SOH}$ estimation is not only an important evidence for calculating SOC, battery equalization and other key parameters, but also of great reference significance regarding necessity of battery replacement, feasibility of degraded use, utilization value evaluation after degradation, and so on. However, the degradation process of lithiumion batteries is a typical nonlinear electrochemical system mechanism, and it is difficult to measure internal chemical characteristic parameters; consequently, estimating $\mathrm{SOH}$ correctly is sufficiently challenging. Currently, this problem has been widely concerned by experts and scholars from all levels including national departments related to new energy vehicles, car manufacturers and the academia.

As an internal intrinsic property of batteries in essence, $\mathrm{SOH}$ belongs to internal invisible state parameters of

Manuscript received January 10, 2019.

Manuscript revised March 7, 2019.

Manuscript publicized March 29, 2019.

${ }^{\dagger}$ The authors are with School of Electrical Engineering and Automation, Harbin Institute of Technology, Harbin, China.

${ }^{\dagger \dagger}$ The author is with Shenzhen Academy of Metrology \& Quality Inspection, Shenzhen, China.

${ }^{\dagger}+$ The author is with School of Science, Harbin University of Science and Technology, Harbin, China.

a) E-mail: zhoudi@smq.com.cn

DOI: 10.1587/transinf.2019EDP7010 batteries that cannot be measured directly [1]. The estimation is to evaluate the degradation levels of battery performance by estimating the current battery performance state. The initial SOH for factory-fresh batteries can be defined as $100 \%$, and the $\mathrm{SOH}$ gradually declines with increasing number of charge-discharge cycles and time of use. Currently, the definition of $\mathrm{SOH}$ has not been completely unified. According to literature, SOH estimations are usually conducted through internal resistance or impedance, capacity or energy, charge-discharge rate, number of cycles and other parameters. The estimation criteria can be a single parameter or a combination of multiple parameters.

Currently, domestic and international research on $\mathrm{SOH}$ of batteries are mainly from the following three aspects [1], [2].

\subsection{Electrochemical Models}

The electrochemical model studies the electrochemical rule of battery capacity degradation from the electrochemical reaction mechanism of batteries, which means to analyze the battery operation mechanism and establish a battery degradation model based on the change rule of kinetic parameters, mass transfer process, thermodynamic properties, mechanical, thermal and electrical properties of materials and other parameters from the perspective of the internal physicochemical process of batteries. The $\mathrm{SOH}$ and remaining life of batteries can be estimated accordingly. The main problem is the large number of model parameters, making it difficult to be put into application. In addition, the process of tests aiming at aging factors are too complex so that there are considerable difficulties in setting up a complete degradation model [3]-[5].

\subsection{Equivalent Circuit Models}

From the perspective of electrotechnology, equivalent circuit models provide equivalent or approximate description of the dynamic property and capacity degradation property of batteries by making lithium-ion batteries be equivalent into circuit models constituting of electrical components according to analysis of large amount of state data from experts with specialized knowledge. This type of models also includes models based on equivalent circuits and semiempirical models based on capacity loss. Compared with electrochemical models, building equivalent circuit models for batteries is more feasible; however, in the approximation 
process, some implication relations between parameters inside batteries that are decisive to system properties may be overlooked, resulting in weak comprehensive ability of equivalent circuit models in describing dynamic and static properties of batteries [6]-[10].

\subsection{Data-Based Analysis Models}

With a large amount of experimental data accumulated in the use of batteries, data-based analysis models summarize an empirical law of battery capacity degradation by statistically estimating the battery $\mathrm{SOH}$ according to analysis of experimental data. This approach draws high attention in the industry because the systematic aging mechanism analysis of batteries is not required in this approach. Data-driven methods often estimate key degradation information from the test data by a specific learning algorithm including as AR (auto regressive models) and GRP (Gaussian process regression). Nuhic et al. proposed SVM approach to construct the aging model of lithium-ion batteries for $\mathrm{SOH}$ Prediction. Besides the kernel techniques, neural network and deep learning techniques were also employed to estimate the $\mathrm{SOH}$ of litium-ion batteries [11]-[18]. Nonetheless, there also exist limitations in the data-driven $\mathrm{SOH}$ estimation due to inevitable errors generated by uncertainty and incompleteness of drive data obtained at the early stage.

It is explicitly stipulated in IEEE Std 1188-1996 that under the standard condition, the SOH of a battery can be determined when the actual battery capacity is lower than $80 \%$ of the rated capacity, indicating that the battery needs to be replaced. The reason is that electric vehicles use battery cell packs to satisfy their capacity needs. When the battery capacity is reduced to $80 \%$, its power can generally still meet the output requirements of an electric vehicle. The $\mathrm{SOH}$ of an EV lithium-ion battery is defined as

$$
S O H=\frac{C}{C_{0}} \times 100 \%
$$

Specifically, $C$ is the EV battery capacity currently measured, and $C_{0}$ is the initial discharge capacity of the EV battery.

In the daily use of electric vehicles, batteries are not recharged after they completely run out, and the initial charge stage is usually between SOC (20-40) \%. A complete battery can be estimated with available capacity because of the inaccuracy of SOC generated by the nonlinearity of the battery capacity and battery degradation. This paper puts forward a battery $\mathrm{SOH}$ estimation method based on three-dimensional fragment charge data of battery voltage $(U)$, charge time $(T)$ and number of charge cycles $(N)$. Different from the research method based on SOC-Uocv, this approach takes absolute charge time as a parameter to avoid influence of SOC value drifts on the online SOH estimation for lithium-ion batteries. The iterated extended Kalman filter-Gaussian process regression (GPR-EKF) is applied to incorporate data at the macro time scale and micro time scale, and kernel function GPR (Gaussian process regression) based on the integration of neutral network and cycles is employed to conduct fitting for the macro time scale data of batteries to determine colored measurement noise; in addition, fragment charge data at the micro time scale is adjusted with real-time iteration to be used as the state equation, which effectively addresses issues of realtime SOC calibration and nonlinearization. Thereby, the online estimation accuracy of SOH is improved.

\section{Principles of an Iterated GPR-EKF Model}

With the different working conditions of electric vehicles, the battery discharge process is very complex and changeable, whereas the charging process is relatively simple and steady. Moreover, since the coulomb efficiency of lithiumion batteries is very high, the charging capacity can be approximated to real capacity of batteries. According to charging mode, the charging capacity is divided into two parts: one is abtained under the constant current charging stage, and the other is abtained under the constant voltage charging stage. In the case of standard charging process, the constant voltage charging capacity of a single lithium-ion battery only accounts for about $2 \%$ of the total capacity of the lithium-ion battery. Moreover, the technology to control the constant voltage charging of the lithium-ion battery is relatively complex. At the same time, overcharging of the lithium ion battery will reduce its life. For the above reasons, only constant current charging is usually performed on electric vehicles and is considered in this paper.

Common external physical parameters characterizing battery working status include voltage, internal resistance and capacity. According to the charge feature of electric vehicles, voltage representations of batteries in different health states vary when they are charged with certain currents in the constant-current charge state, which can be used to characterize the degradation of battery capacity parameter. It is known that an established model of a lithium-ion battery pack is fixed, and empirical lab data of some parameters needs to be utilized as reference. Therefore, the estimation accuracy under different working conditions will be reduced. In order to improve the versatility and adaptability of the model, a real-time iterated model based on actual battery performance should be set up according to historical charge data to complete the estimation model in real time for making the model closer to current working status of the battery pack and improving the estimation accuracy.

Under different health status, charge voltage curves share similarities with basically the same trends. But the curvature of charge curves shows an obvious increase at the later stage. Charge capacity tests of an electric bus battery were carried out on December $6^{\text {th }}, 2013$, May $9^{\text {th }}, 2016$ and October $29^{\text {th }}, 2017$ respectively after its delivery. The relation curves between the charge voltage and time is as illustrated in Fig. 1. Fitting of voltage model curves under different $\mathrm{SOH}$ cannot be performed accurately if the model simply translates and transforms through voltage curves.

Employing a combined estimation technology of state 


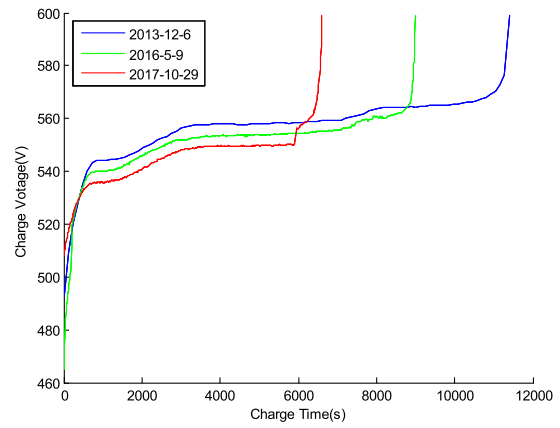

Fig. 1 Full-charge capacity tests for EV batteries at different time

and parameters, the current battery state-of-health estimation lacks adaptability and accuracy. The main reason is that end point voltage of batteries is the only measurable data, but test results indicate that the relation between single capacity and battery voltage changes with the increasing number of charge, making it difficult to establish a determined relation expression of end point voltage and time. In addition, the single SOC value having positive correlation with battery capacity is inaccurate due to battery capacity degradation and errors generated from capacity estimation using fragment data are accumulated, resulting in failure in convergence. Based on the combination of Kalman filter and Gaussian process regression, the multiple-time-scale model is integrated. To ensure the micro model is gradually iterated as the number of charge increases, the macro data model will be utilized as the iterative convergence condition for the micro data model for guaranteeing the accuracy of battery $\mathrm{SOH}$ estimation.

In actual research, main studies of the Kalman filter focus on the nonlinear system due to the nature of the objective world, nonlinearity. The Kalman filter is the minimum variance estimation designed in time domain based on the least square method.

The following nonlinear discrete state space model is considered:

$$
\left\{\begin{array}{c}
x_{k}=f\left(x_{k-1}\right)+\omega_{k-1} \\
z_{k}=h\left(x_{k}\right)+v_{k}
\end{array}\right.
$$

Specifically, the state vector $x_{k} \in \mathrm{R}^{\mathrm{n}}$ is unobservable, and $z_{k} \in \mathrm{R}^{\mathrm{m}}$ is observable measurement vector; $f(\bullet)$ and $h(\bullet)$ are known nonlinear functions; process noise $\omega_{k-1} \in \mathrm{R}^{\mathrm{n}}$ and measurement noise $\gamma_{k} \in \mathrm{R}^{\mathrm{m}}$ are assumed to be mutually independent Gaussian noises by adopting the suboptimal estimation method, whose mean values are $w_{k-1}$ and $v_{k}$ respectively and covariance matrixes are $Q_{k-1}$ and $R_{k}$ respectively.

Gaussian process regression (GPR) provides a selfconsistent and practical probabilistic method to address the core issue in the machine learning field, attracting much attention from scholars. Machine learning is related to statistical technologies. Current methods for massive data learning include support vector machine (SVM), neutral network, relevance vector machine, and so on. Rasmussen proposes a Gaussian process algorithm to be applied in the machine learning field [19]. Meanwhile, the appearance of kernel functions and their successful application enable an increasing number of people to introduce kernel functions into Gaussian processes [20], [21], where the covariance function of a Gaussian process is its kernel function. Gaussian processes (GP) is mainly adopted in two supervised learning problems, regression and classification. Gaussian processes have obtained certain achievements on time series prediction in recent years [22], [23]. Robert et al. applied Gaussian processes in the modelling for time series such as stock forecasting and so on [24].

In making Gaussian process regression forecast, the mean function is denoted as $m(x)$, the covariance function is denoted as $k\left(x, x^{\prime}\right)$, and they are defined as the follows:

$$
\begin{aligned}
& m(x)=\mathrm{E}(f(x)) \\
& k\left(x, x^{\prime}\right)=\mathrm{E}[f(x)-m(x)]\left[f\left(x^{\prime}\right)-m\left(x^{\prime}\right)\right]
\end{aligned}
$$

Consider a simple regression model:

$$
y=f(x)+\varepsilon \quad f(x)=X^{T} w
$$

The training set is $\left\{\left(x_{i}, y_{i}\right) \mid i=1, \ldots, n\right\}$, where $x_{i}(i=$ $1, \ldots, n)$ is the input value, $f$ is the function value, $y_{i}(i=$ $1, \ldots, n)$ is the output value; $\varepsilon$ represents independent and identically distributed white Gaussian noise whose mean value is 0 and variance is $\sigma^{2}$. The following form can be obtained: $\varepsilon=\left(0, \sigma^{2}\right)$.

The probability density function of the observed value $y$ is obtained as follows:

$$
\begin{aligned}
p(y \mid X, w) & =\prod_{i=1}^{n} p\left(y_{i} \mid x_{i}, w\right) \\
& =\prod_{i=1}^{n} \frac{1}{\sqrt{2 \pi} \sigma_{n}} \exp \left(-\frac{\left(y_{i}-x_{i}^{T} w\right)^{2}}{2 \sigma_{n}^{2}}\right) \\
& =\frac{1}{\left(2 \pi \sigma_{n}^{2}\right)^{n / 2}} \exp \left(-\frac{1}{2 \sigma_{n}^{2}}\left|y-X^{T} w\right|^{2}\right) \\
& =N\left(X^{T} w, \sigma_{n}^{2} I\right)
\end{aligned}
$$

Thus, the prior distribution of the observed value $y$ is:

$$
y \mid X, w=N\left(X^{T} w, k\left(x, x^{\prime}\right) \mid \sigma^{2} I\right)
$$

When estimation of predicted value is applied, the distribution of the observed value $y$ and the predicted value $f_{*}$ is as follows:

$$
\left(\begin{array}{c}
y \\
f_{*}
\end{array}\right)=N\left(0,\left(\begin{array}{cc}
k(X, X) & k\left(X, x_{*}\right) \\
k\left(x_{*}, X\right) & k\left(x_{*}, x_{*}\right)
\end{array}\right)\right)
$$

Specifically, $k\left(X, x_{*}\right)$ represents covariance between training data and predicted values, and $k\left(x_{*}, x_{*}\right)$ is covariance between predicted values.

When the observed value contains noise, the covariance of the observed value is $\operatorname{cov}(y)=K(X, X)+\sigma_{n}^{2} I$, the joint distribution of the observed value and the test value is:

$$
\left(\begin{array}{c}
y \\
f_{*}
\end{array}\right)=N\left(0,\left(\begin{array}{cc}
K(X, X)+\sigma_{n}^{2} I & K\left(X, X_{*}\right) \\
K\left(X_{*}, X\right) & K\left(X_{*}, X_{*}\right)
\end{array}\right)\right)
$$


The posteriori distribution of weights can be obtained according to Bayesian inference. The posterior distribution inference of the Bayesian linear model is as follows:

$$
\begin{aligned}
f_{*} \mid X, f, x_{*}= & N\left(k\left(x_{*}, X\right) k(X, X)^{-1} f, k\left(x_{*}, x_{*}\right)\right. \\
& \left.-k\left(x_{*}, X\right) k(X, X)^{-1} k\left(X, x_{*}\right)\right)
\end{aligned}
$$

Currently, there is no unified theoretical foundation of kernel function type selection for Gaussian processes. Therefore, this paper aims to determine the kernel function type through influences of single and combining kernel functions on distribution of prediction as well as tests of a massive amount of data.

For kernel function multiplication, when two kernel functions are mutually independent, $k\left(x, x^{\prime}\right)=k_{1}\left(x, x^{\prime}\right)$ $k_{2}\left(x, x^{\prime}\right)$, the product of two kernel functions are still a kernel function, and $k_{1}\left(x, x^{\prime}\right)$ is considered as a structural modification for the other kernel function $k_{2}\left(x, x^{\prime}\right)$. A single kernel function usually only describes one aspect of data characteristics, but it is difficult for a GPR model based on a single kernel function to provide accurate description for a highly complex system. Therefore, multiple kernel functions can be combined to describe heterogeneous information in data for enhancing the nonlinear mapping ability of kernel functions.

EKF is the prior knowledge (mainly covariance information) based on decided system models (including system state models and measurement models) and system noises. However, in many cases, it is difficult to obtain system models or accurate system models and statistical properties of system noises are also unknown, leading to sharp decline in filter algorithm effectiveness or even being unable to work. This paper integrates the GPR method into EKF to use the GPR method for identifying system models, including state transition models and measurement models as well as the corresponding covariance matrixes of noises, in order to substitute or enhance original system models. It can solve the state estimation problem in the case of unknown system model or inaccurate system model. Establishing a parameterized model requires a large amount of specialized knowledge. Some systems can only set up simplified models, and it is difficult to comprehensively characterize actual systems with these parameterized models. GPR models can overcome deficiencies in using parameterized models by conducting multiple-dimensional data training to improve model accuracy, because GPR models can characterize system information that parameterized models fail to capture. Besides, the generalization performance is also enhanced. In essence, GPR models characterize residual output of the system in addition to contribution of separation parameter models.

Regarding different moment $k$, iterated GPR-EKF is described as follows by a nonlinear system consisting of a random variable $x$ with Gaussian noise $W(k)$ and an observable variable $z$ with Gaussian noise $V(k)$.

$$
\begin{aligned}
& x_{k}=G P^{f}\left(x_{1}, x_{2} \ldots x_{k-1}\right)+W(k) \\
& z_{k}=G P^{h}\left(x_{k}\right)+V(k)
\end{aligned}
$$

Specifically, $G P^{f}$ is a nonlinear state equation function based on GPR; $G P^{h}$ is a nonlinear measurement equation function based on GPR. In the traditional Kalman algorithm, prediction of state can be obtained with only one calculation step by substituting state of last moment into the state equation. However, iterated GPR-EKF utilizes original $x_{1}, x_{2} \ldots, x_{k-1}$ here to predict $x_{k}$, and calculates their weights for the mean value to obtain one-step-ahead prediction of system state. After hyper parameters of GPR are optimized by learning training data, obtained state transition GPR models and measurement GPR models can be used to substitute system models to gain iterated GPR-EKF algorithm.

\section{Realization of Battery State-of-Health Models}

It is discussed previously that it is unable to conduct full charge and discharge for lithium-ion batteries in daily charge; thus, the definite actual available charge capacity of lithium-ion batteries cannot be gained directly. The algorithm proposed in this paper integrates Gaussian process regression into the Kalman filter to establish an iterated GPREKF model, and fragment charge data based on the micro time scale iterates the nonparametric measurement function in the Kalman filter $\left(G P_{k}^{f}\right)$. Meanwhile, the empirical data based on the macro time scale is combined with the historical charge data to establish colored measurement noise with a nonzero expected value, whose mean value is $v_{k}$ and covariance matrix is $R_{k}$.

Determine related parameters of the initial battery cycle loop $_{0}$, constant-current charge current $I$, cut-off voltage of constant-voltage charge $U$, full-charge data in the initial constant-current charge $d_{0}=\left(t_{0}(n), U_{0}(n)\right), k=1,2, \ldots, n_{0}$, $n_{0}$ is total number of sampling time points when the battery reaches the cut-off voltage $V$ in the charge with constant current $I . t_{0}(n)$ is the discrete relative time of the equivalent interval sampling, and the sampling time interval $T_{\mathrm{s}}=t_{0}(n+1)-t_{0}(n)$ is a constant. $v_{0}(n)$ represents the voltage of the sampling point $k$. The least square method is applied to conduct fitting for initial data, whose measurement function is $G P_{0}^{f}$.

The $k$ th daily charge fragment data is $d_{k}=\left(t_{k}(n), v_{k}(n)\right)$, $n=1,2, \ldots, n_{k}$. Because the absolute time of fragment data time starts from 0 , the overall charge data is sorted here. The final stop time of charging is as 0 and the start time of charging is as $\left(n_{k}-1\right) T_{\mathrm{s}}$, which is conductive to the subsequent collation of prediction data (as shown in Fig. 2).

$$
t_{k}=0, T_{s}, 2 T_{s}, 3 T_{s} \cdots\left(n_{k}-1\right) T_{s}
$$

Conduct EKF-GPR for the $k$ th fragment data, estimate required full-charge time for the $k$ th constant-current charge, and establish a nonparametric iterated GPR-EKF model:

$$
U_{n}^{k}=G P_{k}^{f}\left(U_{1}^{k}, U_{2}^{k}, \ldots U_{n-1}^{k}\right)+W_{n-1}^{k}
$$

Measurement equation:

$$
t_{n}^{k}=G P^{h}\left(U_{n}^{k}\right)+V_{n}^{k}
$$




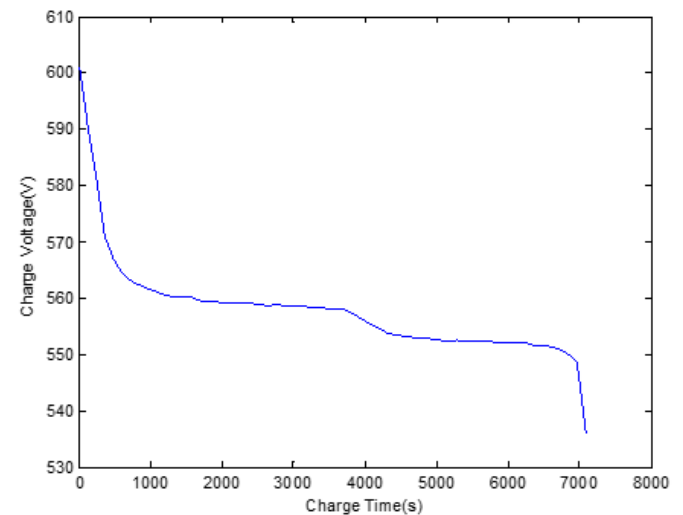

Fig. 2 Fragment charge data after adjustment

Where $U_{n}^{k}$ : measured charge voltage value at the charge moment; $t_{n}^{k}$ : charge moment.

$G P_{k}^{f}$ is gained as the first state equation function of $G P_{0}^{f}$ according to the first Gaussian process regression of the full-charge curve. Meanwhile, iterative prediction of voltage points except for fragment data is made according to the prediction characteristic of Gaussian process, which means to predict $U_{n}^{k}$ according to voltage values of $U_{1}^{k}, U_{2}^{k} \cdots U_{n}^{k}$. After implementing the Kalman filter, modified $U_{n}^{k}$ and previous voltage values $U_{1}^{k}, U_{2}^{k} \cdots U_{n}^{k}$ are utilized to predict the voltage value of the next point $U_{n+1}^{k}$.

Along with the increasing time of use, the EV battery capacity and charge curve characteristics change gradually. Therefore, shifts will occur gradually in the constant adoption of nonparametric GPR models based on initial fullcharge data, leading to estimation result drifts. Suppose colored measurement noise is $V_{k}$ Gaussian noise. Make a prediction about the macro battery capacity degradation data by applying Gaussian process regression of the covariance function of product of neutral network covariance function and periodic kernel function, and the covariance of measurement noise $R_{k}$ is obtained.

$k_{1}\left(x, x^{\prime}\right)$ is set to be a neutral network kernel function, a local kernel function, to describe the nonlinear characteristics of data. $k_{2}\left(x, x^{\prime}\right)$ is set to be a periodic kernel function, a global kernel function, to describe periodic characteristics of data. Expressions of $k_{1}\left(x, x^{\prime}\right)$ and $k_{2}\left(x, x^{\prime}\right)$ are

$$
\begin{aligned}
& k_{1}\left(x, x^{\prime}\right)=\sigma_{f}^{2} \sin ^{-1}\left(\frac{x^{T} \Lambda^{-2} x^{\prime}}{\sqrt{\left(1+x^{T} \Lambda^{-2} x\right)\left(1+x^{\prime} \Lambda^{-2} x^{\prime}\right)}}\right) \\
& k_{2}\left(x, x^{\prime}\right)=\sigma_{f}^{2} \exp \left(-\frac{2}{l^{2}} \sin ^{2} \frac{\pi\left\|x-x^{\prime}\right\|}{p}\right)
\end{aligned}
$$

Steps for initializing hyper parameters in mean value functions and covariance functions of GPR prediction models include that hyper parameter in mean value function and covariance function of a GPR prediction model is denoted as $\Theta$, and the hyper parameter is initialized as $\Theta=$ $[a, b, 1, \mathrm{sf} 1, \mathrm{ell}, \mathrm{p}, \mathrm{sf} 2]^{T}$.

Further, the logarithmic maximum likelihood estimation function is:

$$
\begin{aligned}
& \log p(y \mid X)=-\frac{1}{2} y^{T}\left(K+\sigma^{2} I\right)^{-1} y \\
& -\frac{1}{2} \log \left|K+\sigma^{2} I\right|-\frac{n}{2} \log 2 \pi
\end{aligned}
$$

Iterated GPR-EKF equations are as follows:

Prediction:

$$
\begin{aligned}
& U(n \mid n-1)=G P^{f}(U(n-1 \mid n-1)) \\
& \widehat{P}(n \mid n-1)=P(n-1 \mid n-1)+Q_{k}
\end{aligned}
$$

Update model, use difference quotient to approximate the derivative:

$$
\begin{aligned}
\hat{H}(n \mid n-1)= & \left(G P^{h}\left(U_{n-1}^{k}\right)-G P^{h}\left(U_{n}^{k}\right)\right) \\
& /\left(U_{n-1}^{k}-U_{n}^{k}\right)
\end{aligned}
$$

Calculate gain:

$$
\begin{aligned}
K(n)= & \hat{P}(n \mid n-1) H^{T}(n) \\
& {\left[\hat{H}(n) P(n \mid n-1) H^{T}(n)\right.} \\
& +R(n)]^{-1}
\end{aligned}
$$

Update state:

$$
\begin{aligned}
\hat{x}(n \mid n)= & x(n \mid n-1)+K(n) \\
& {\left[z(n)-v_{k}-G P(x(n \mid n-1))\right] }
\end{aligned}
$$

Update covariance:

$$
\hat{P}(n \mid n)=[I-K(n) H(n)] P(n \mid n-1)
$$

Finally, the absolute moment corresponding to the initial voltage $U$, required full-charge time for the $k$ th charge $T_{k}$, and available capacity for the $k$ th constant-current charge are gained as following

$$
C_{k}=t_{k} \times I
$$

where $I$ is the current value of constant-current charge of the battery.

Finally, the real-time battery SOH estimation result is obtained.

$$
S O H=\frac{C_{k}}{C_{0}}
$$

Where $C_{k}$ is the available capacity for the $k$ th constant current charge; $C_{0}$ is the initial constant-current charge capacity.

\section{Model Validation and Analysis}

The online estimation model of battery state-of-health algorithm was validated based on battery testing data and daily charge data of an electric bus. Figure 3 is a picture from the battery test scene. The selected test equipment is a battery charge and discharge machine manufactured by Sin excel, whose technical specifications of voltage accuracy and current accuracy are $0.5 \%$ and $1.0 \%$ respectively. To evaluate $\mathrm{SOH}$ of electric bus power batteries, the battery pack is 


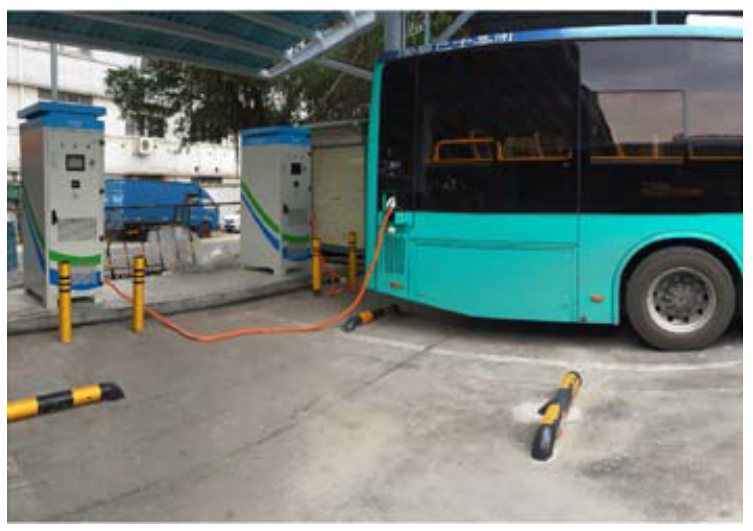

Fig. 3 Scene of electric bus battery test

Table 1 Specifications of the battery pack.

\begin{tabular}{ll}
\hline Item & Specification \\
The number of modules & 63 \\
Compound mode of modules & A battery pack consists three \\
parallel branches each of which & $\begin{array}{l}\text { is comprised of twenty-one } \\
\text { modules form a branch in series. }\end{array}$ \\
& $540 \mathrm{Ah}$ \\
Nominal Capacity & $537.6 \mathrm{~V}$ \\
Nominal Voltage & $336 \mathrm{~V} \sim 638 \mathrm{~V}$ \\
$\begin{array}{l}\text { Working Voltage } \\
\text { Charging Cut-off Voltage of single } \\
\text { batteries }\end{array}$ & $3.8 \mathrm{~V}$ \\
$\begin{array}{l}\text { Disharging Cut-off Voltage } \\
\text { single batteries }\end{array}$ & $2.0 \mathrm{~V}$ \\
$\begin{array}{l}\text { Nominal charge current } \\
\text { Max. charge current }\end{array}$ & $100 \mathrm{~A}$ \\
$\begin{array}{l}\text { Max. discharge current } \\
\text { Operating Temperature }\end{array}$ & $200 \mathrm{~A}$ \\
& $100 \mathrm{~A}$ \\
\hline
\end{tabular}

composed of sixty-three battery modules (basic units). Each module consists of 8 single batteries in series. All modules are divided into three same branches in parallel where the twenty-one modules within each branches are connected in series. The specifications of the battery pack are summarized in Table 1.

The first full-charge capacity test of the electric bus was conducted after its delivery on December $6^{\text {th }}, 2013$. The iterated GPR-EKF battery state-of-health estimation model was applied based on full-charge data and historical charge data to provide online battery state-of-health estimation for fragment charge data of March $10^{\text {th }}, 2014$. The initial battery SOC reading before charging was $36 \%$. Figure 4 shows the full-charge curve of battery on December $3^{\text {rd }}, 2013$ and estimated battery curve of March $3^{\text {rd }}, 2014$. According to online estimation, the final available charge capacity of the bus battery on March $10^{\text {th }}, 2014$ was

$$
C_{k}=t_{k} \times I=550.6 \mathrm{Ah}
$$

where $I$ stands for constant current of charging process.

Accordingly, the state of health of the battery at that time was

$$
S O H=\frac{C_{k}}{C_{0}}=0.979
$$

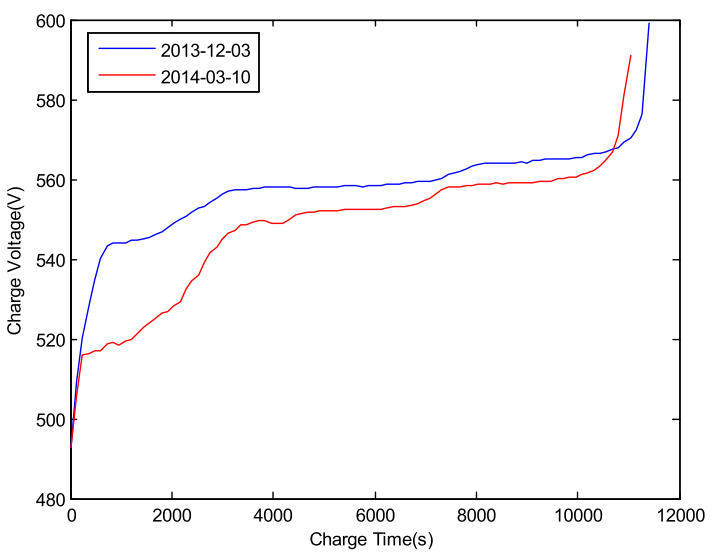

Fig. 4 Comparison of estimated curve and initial full-charge curve of the battery

Table 2 The actual measured results of available capacity

\begin{tabular}{ll}
\hline Date of full charge test & Test result \\
December $6^{\text {th }}, 2013$ & $562.3 \mathrm{Ah}$ \\
May $9^{\text {th }}, 2016$ & $522.1 \mathrm{Ah}$ \\
October $29^{\text {th }}, 2017$ & $472.8 \mathrm{Ah}$ \\
\hline
\end{tabular}

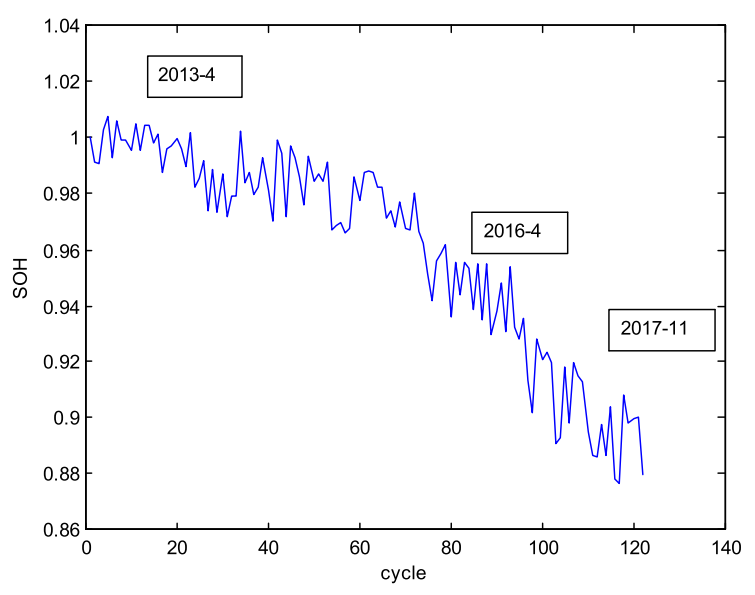

Fig.5 Results of the battery state-of-health estimation

Three full-charge battery tests were carried out for the electric bus successively from 2013 to 2017 . The full process curve of charge is as shown in Fig. 4. The actual measured results of available capacity are as shown in Table 2.

Historical charge data of the electric bus with the SOC initially lower than $40 \%$ being charged to $100 \%$ was selected to perform online battery estimation. 122 sets of fragment charge data from 2013 to 2017 were chosen to conduct data verification. Figure 5 shows results of the battery stateof-health estimation.

The estimated charge capacity data of the electric bus fragment charge data with the full-charge battery test interval within 3 days was selected for comparison.

The accuracy of battery charge capacity cannot be reproduced because of the battery capacity fluctuation. The degradation level of battery capacity does not change suddenly under normal working condition of the battery, and the 
Table 3 The comparison of estimated charge capacity data

\begin{tabular}{lllll}
\hline Date of charge & $\begin{array}{l}\text { Initial } \\
\text { SOC }\end{array}$ & $\begin{array}{l}\text { Final } \\
\text { SOC }\end{array}$ & $\begin{array}{l}\text { Estimated } \\
\text { capacity }(\mathrm{Ah})\end{array}$ & $\begin{array}{l}\text { Capacity } \\
\text { fluctuation }\end{array}$ \\
December $8^{\text {th }}, 2013$ & $32 \%$ & $100 \%$ & 557.9 & $-0.78 \%$ \\
May $8^{\text {th }}, 2016$ & $22 \%$ & $100 \%$ & 537.4 & $2.93 \%$ \\
November $1^{\text {st }}, 2017$ & $35 \%$ & $100 \%$ & 498.5 & $5.43 \%$ \\
\hline
\end{tabular}

capacity fluctuation of a fully-functioning battery is usually lower than $2 \%$. Comparing estimated data with the actual data, the capacity fluctuation is lower than $6 \%$; accordingly, it can be preliminarily estimated that the accuracy of capacity estimation should be better than $8 \%$.

In order to better evaluate $\mathrm{SOH}$ of batteries and ensure the safety of use, the numerous charging data and corresponding SOH information of batteries in the same type can be introduced to analysis accuracy and uncertainty of estimation based on clustering method. K-means method is used to cluster historical data under different working conditions. Then the accuracy and uncertainty of SOH estimation can be evaluated by calculating the distances between estimated data and cluster centers. If the given data is an outiliers (far away from all cluster centers), it shows that the $\mathrm{SOH}$ estimation is perhaps not accurate enough. Because, at present, full charge/discharge testing is still the most stable and reliable method for evaluating $\mathrm{SOH}$ of batteries, it is necessary to conduct a full charging test to obtain relatively accurate $\mathrm{SOH}$ measure for updating the initial state of iteration and training data. If these two estimated results are in agreement, the accuracy of the estimation will be further illustrated and the battery itself has problems. Conversely, the accuracy and robustness of the estimation can be improved by correction based on full charge/discharge process.

\section{Conclusion}

Based on the charge data of electric vehicles under the normal working condition, the online state-of-health estimation method for in-use electric vehicle batteries designed in the paper can monitor the state of health of batteries in real time, which is conductive to the recycling of batteries and effective improvement of battery maintenance level. Estimating the full charge time of batteries by using fragment data is the main function of the model. The iterated GPR-EKF algorithm proposed in the paper firstly integrates Gaussian process regression with the extended Kalman filter to approximate the state equation, measurement equation and noise of EKF before utilizing the difference quotient to approximate the state matrix and the measurement matrix. The online SOH estimation error can be controlled within $8 \%$ according to the data results of actual electric vehicle battery tests and battery charge during the full life cycle.

\section{Acknowledgements}

This work was supported by Science and Technology Innovation Committee of Shenzhen Municipality No. JSGG20170824094640025.

\section{References}

[1] C. Lin, A. Tang, and W. Wang, "A review of SOH estimation methods in Lithium-ion batteries for electric vehicle applications," Science Direct, pp.1920-1925, 2015.

[2] V. Srinivasan and J. Newman, "Discharge model for the lithium iron-phosphate electrode," Journal of the Electrochemical Society, vol.151, no.10, pp.A1517-A1529, 2004.

[3] B. Saha, K. Goebel, and J. Christophersen, "Comparison of prognostic algorithms for estimating remaining useful life of batteries," Transactions of the Institute of Measurement and Control, vol.31, no.3-4, pp.293-308, 2009.

[4] J.C. Forman, S.J. Moura, J.L. Stein, and H.K. Fathy, "Genetic identification and fisher identifiability analysis of the Doyle-Fuller-Newman model from experimental cycling of a LiFePO4 cell," Journal of Power Sources, vol.210, pp.263-275, 2012.

[5] R. Ahmed, M.E. Sayed, H. Arasaratnam, and S. Habibi, "Reducedorder electrochemical model parameters identification and SOC estimation for healthy and aged Li-ion batteries," Emerging and Selected Topics in Power Electronics, vol.2, no.3, pp.659-677, 2014.

[6] A. Allam and S. Onori, "Characterization of aging propagation in Lithium-ion cells based on an electrochemical model," American Control Conference, pp.3113-3118, 2016.

[7] Z. Chen, C.C. Mi, Y. Fu, J. Xu, and X. Gong, "Online battery state of health estimation based on Genetic Algorithm for electric and hybrid vehicle applications," Journal of Power Sources, vol.240, pp.184-192, 2013.

[8] H. Chaoui, N. Golon, I. Hmouz, et al., "Lyapunov-based adaptive state of charge and state of health estimation for Li-ion batteries," IEEE Trans. Ind. Electron., vol.62, no.3, pp.1610-161, 2015.

[9] N. Omar, M.A. Monem, Y. Firouz, J. Salminen, J. Smekens, O. Hegazy, H. Gaulous, G. Mulder, P.V. Bossche, T. Coosemans, and J. Van Mierlo, "Lithium iron phosphate based battery-Assessment of the aging parameters and development of cycle life model," Applied Energy, vol.113, pp.1575-1585, 2014.

[10] C. Weng, X. Feng, J. Sun, and H. Peng, "State-of-health monitoring of lithium-ion battery modules and packs via incremental capacity peak tracking," Applied Energy, vol.180, pp.360-368, 2016.

[11] D. Liu, Y. Luo, J. Liu, Y. Peng, L. Guo, and M. Pecht, "Lithium-ion battery remaining useful life estimation based on fusion nonlinear degradation AR model and RPF algorithm," Neural Computing and Applications, vol.25, no.3-4, pp.557-572, 2014.

[12] C. Hu, G. Jain, P. Zhang, C. Schmidt, P. Gomadam, and T. Gorka, "Data-driven method based on particle swarm optimization and knearest neighbor regression for estimating capacity of lithium-ion battery," Applied Energy, vol.129, pp.49-55, 2014.

[13] H.-T. Lin, T.-J. Liang, and S.-M. Chen, "Estimation of Battery State of Health Using Probabilistic Neural Network," IEEE Trans. Ind. Informat., vol.9, no.2, pp.679-685, 2013.

[14] D. Andre, A. Nuhic, T. Soczka-Guth, and D.U. Sauer, "Comparative study of a structured neural network and an extended Kalman filter for state of health determination of lithium-ion batteries in hybrid electric vehicles," Engineering Applications of Artificial Intelligence, vol.26, no.3, pp.951-961, 2013.

[15] J. Zhou, Z. He, M. Gao, and Y. Liu, "Battery state of health estimation using the generalized regression neural network," International Congress on Image and Signal Processing, pp.1396-1400, 2016.

[16] G. Shi, "Mining and knowledge discovery for geoscientists," Support Vector Machines, ch.4, pp.87-110, Oxford Elsevier, 2014.

[17] H. Zhang, Q. Miao, X. Zhang, and Z. Liu, "Remaining useful life prediction of lithium-ion battery with unscented particle filter technique," Microelectronics Reliability, vol.53, no.6, pp.805-810, 2013.

[18] S. Tang, C. Yu, X. Wang, X. Guo, and X. Si, "Remaining useful life prediction of lithium-ion batteries based on the wiener process with measurement error," Energies, vol.7, no.2, pp.520-547, 2014. 
[19] C.E. Rasmussen, "Evaluation of Gaussian processes and other methods for non-linear regression," Ph.D. thesis, University of Toronto, 1996.

[20] C.E. Rasmussen and C.K.I. Williams, Gaussian Processes for Machine Learning, Springer, 2006.

[21] C.E. Rasmussen, "Advanced Lectures on Machine Learning," in Gaussian Processes in Machine Learning, pp.63-71, Springer, Berlin Heidelberg, 2004.

[22] S. Brahim-Belhouari and A. Bermak, "Gaussian process for nonstationary time series prediction," Computational Statistics and Data Analysis, vol.47, no.4, pp.705-712, 2004.

[23] C.K.I. Williams, "Prediction with Gaussian Processes," in Linear Regression to Linear Prediction and Beyond Learning in Graphical Models, pp.599-621, Springer, Netherlands, 1998.

[24] S. Robert, M. Osborne, M. Ebden, etc., "Gaussian processes for time-series modeling," Philosophical Transactions of the Royal Society A: Mathematical, Physical and Engineering Sciences, p.371, 2013.

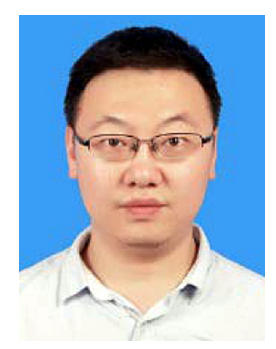

Di Zhou is a PhD Candidate of Harbin Institute of Technology, China. He received his master degree from Harbin Institute of Technology in 2009. His main research interests is health assessment of EV power battery, charging facility measurement and testing technology.

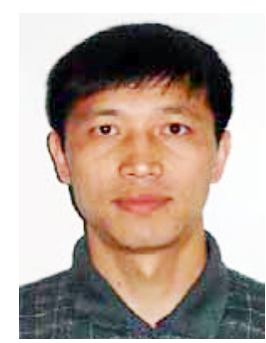

Ping Fu received his Ph.D. degree from Harbin Institute of Technology in 1999. He is now a professor at Harbin Institute of Technology, China. His main research areas are data mining, compressive sensing and automatic test technology and etc.

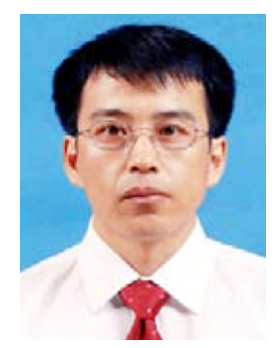

Hongtao Yin received the Ph.D. degree from Harbin Institute of Technology in 2006. He is now a lecturer at Automatic Test and Control Institute, Harbin Institute of Technology. His current research interests include data mining and computer vision.

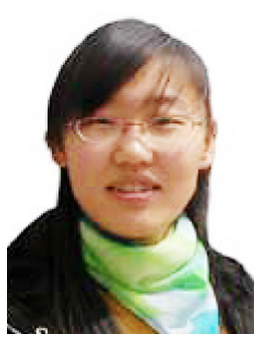

Wei Xie received the Ph.D. degree from Harbin Engineering University in 2014. She is now a lecturer at School of Science, Harbin University of Science and Technology. Her current research interests include image analysis, machine Learning and data mining.

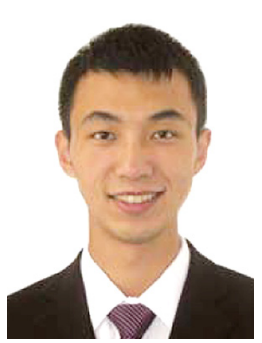

Shou Feng is a PhD Candidate of Harbin Institute of Technology, China. He received his master degree from Harbin Institute of Technology. His main research interests include automatic test and control technology, data mining and etc. 